\title{
An Efficient Signal Acquisition Method for GPS Software-Defined Radio Receivers
}

\author{
Tung Hai Ta
}

NAVIS Centre, Hanoi University of Science and Technology

No. 1 Dai Co Viet, Hanoi. Email: tung.tahai@hust.edu.vn

2 Abstract-With the development of the computational power of programmable processors, the Software-Defined Radio (SDR) approach for GPS receivers has been widely considered. However, in comparison with the conventional hardware receivers, the computational speed of the SDR receivers is still a problem needed to be improved. As a first process of the digital processing part, and also the most resource consuming process of a GPS receiver, the signal acquisition process is very important and needed to be improved in order to suit with the SDR approach. This paper introduces a hybrid signal acquisition method leveraging the conventional serial search acquisition (typical in hardware receivers), and the FFT-based parallel search (typical in SDR receivers) in order to significantly improve the acquisition sensitivity, meanwhile keep the computational complexity still comparable with that of the FFT-based parallel method.

Keywords-GPS, Acquisition, FFT.

\section{INTRODUCTION}

Conventionally, to produce positioning and timing information, a GPS receiver experiences three main stages: signal synchronization; navigation data demodulation; and position, velocity and time computation. The signal synchronization stage is usually divided into signal acquisition and tracking processes. In particular, the signal acquisition is very critical because it is the first operation performed by the receiver. The signal acquisition is performed by correlating the incoming and the local generated signals to measure the similarity between the twos. The local signal is generated based on the uncertainty regions of the code delay, Doppler shift and satellite number (i.e. 3D search-space). If the level of the similarity represented by the correlation values is over a predetermined threshold then the code acquisition stops and transfers the estimated values of satellite number, code delay and Doppler shift to the tracking stage for finer estimation.

As the main part of the acquisition, the correlation process can be performed by using conventional serial search [1] or Fast Fourier Transform (FFT) parallel search [2]. The serial search method tests each cell, whilst the FFT-based parallel test multiple cells of the search-space at a time. The former method is used in conventional hardware receivers due to its simplicity; however for Softwared Defined Radio (SDR) receivers this type is not efficient due to the high computational complexity [3]. On the contrary, the latter method is very efficient for software receivers since it utilises the fast nature of the FFT algorithm; but not suitable for hardware receivers due to the complexity of FFT algorithm. Moreover, the code Doppler effect, which causes sensitivity loss, might reduce the quality of estimation of the FFT with respect to the serial search, when the integration time is large.

In an effort of finding a suitable acquisition method for SDR GPS receivers, this paper introduces a hybrid method which utilises both the serial search and FFT correlators. By using this method, the acquisition stage could inherit the fast computation nature of the FFT; whilst maintain the quality of the estimation as good as the serial search method.

The remaining part of this paper is organised as follow: Section II introduces the fundamentals of signal acquisition including the serial search and FFTbased search. Section III describes the hybrid acquisition method. Section IV analyses the performance of the hybrid method in comparisons with the serial search and FFT parallel methods. Finally, some remarks are drawn at the conclusion section.

\section{GPS L1 C/A Signal ACquisition Process}

\section{A. Signal and acquisition process}

The received GPS L1 C/A signal after the Analog to Digital Converter can be represented as ([4-6])

$$
r[n]=\sqrt{2 C} d[n] c[n+\tau] \cos \left(2 \pi\left(f_{I F}+f_{D}\right) n T_{S}+\varphi\right)+n_{W}[n]
$$


where $C$ is the carrier power (W); $d[n]$ is the navigation data; $c[n]$ is the PRN code which is assigned uniquely to each satellite, $f_{I F}, f_{D}$ denote the Intermediate Frequency (IF) and Doppler shift (Hz) respectively; $T_{S}=1 / F_{S}$ stands for the sampling period (s) $\left(F_{S}\right.$ is the sampling frequency $(\mathrm{Hz}) ; \varphi$ is the initial carrier phase (rad); $\tau$ is the initial code delay (samples) ; and $n_{W}$ is the Additive White Gaussian Noise (AWGN) with zero mean $(\mu=0)$ and variance $\sigma_{n}^{2}\left(n_{W} \sim \mathcal{N}\left(0, \sigma_{n}^{2}\right)\right)$.

As introduced in [1], the conventional acquisition process strives to determine the presence of a desired signal defined by PRN code $(c)$, code delay $(\tau)$ and Doppler offset $\left(f_{d}\right)$ in the incoming signal. The uncertainty regions of $\left(c, \tau, f_{D}\right)$ form a signal search-space, each cell $\left(\hat{c}, \hat{\tau}, \hat{f}_{d}\right)$ of which is used to locally generate an equivalent tentative signal, see Figure 1. In Figure 1, the search-space has the conventional step-size values in the two dimensions as follows [1]:

$$
\triangle f_{D}=\frac{1}{2 T_{c o h}} \mathrm{~Hz} ; \text { and } \triangle \theta=0.5 \text { chip }
$$

with $T_{c o h}$ being the coherent integration time (s). These values together with the uncertainty regions define the size of the search-space, $L=N_{c} \times N_{f}$, where $N_{c}$ is the number of columns (i.e. possible code delays to be tested) and $N_{f}$ is the number of rows (i.e. possible Doppler shifts to be tested). As proposed in [1], in a conventional operational scenario, for the GPS signal acquisition process, $f_{D} \in[-5,5] \mathrm{kHz}$, and $\theta \in[0,1022]$ chip (i.e. one full code period). Therefore, $N_{c}=2046$, $N_{f}=21$, and $L=42,966$ combinations.

The acquisition process correlates the incoming signal $(r[n])$ with the tentative signal $(\hat{r}[n])$ to measure the similarity between the two signals. Basically, there are two acquisition approaches as follows:

- Conventional serial search: the acquisition process tests one cell (i.e. a tentative signal) at a time.

- Parallel search using Fast Fourier Transform (FFT) method: the acquisition process tests multiple cells at a time

- Parallels in code delay dimension: all cells of a whole row of the search space are tested at once.

- Parallels in Doppler shift dimension: all cells of a whole column of the search space are tested at once.

However, since $N_{f}<<N_{c}$, hence the parallel search in Doppler shift dimension is less efficient than that in code delay dimension. Therefore, in this paper, only the parallels in code delay, which is referred to as the FFTtime method from now on, is considered.

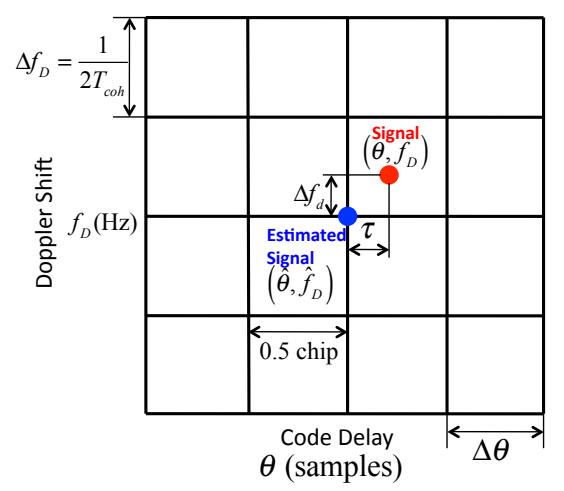

Figugre 1. the search space of a signal acquisition process

\section{B. Serial Search Approach}

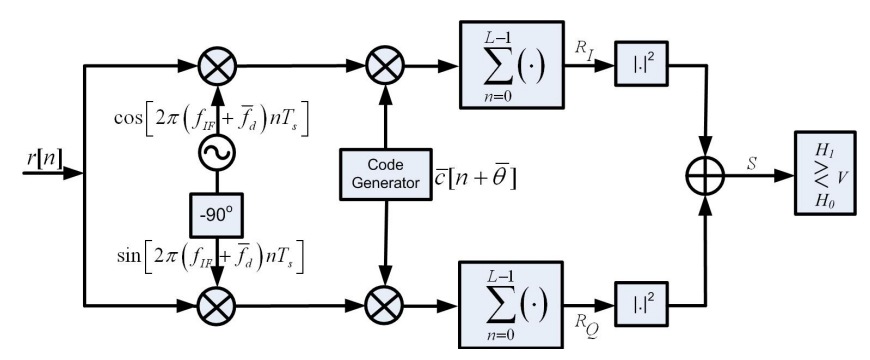

Figugre 2. Conventional serial search acquisition process in a GNSS receiver

In a conventional serial acquisition process as depicted in Figure2, the received signal is multiplied by the local complex tentative signal defined by a single cell in the search-space. Then the results are summed by the accumulator. Consequently, at the accumulator output, the complex-valued correlation $R_{B}$ between the incoming and the local generated signals is obtained in correspondence with the tentative set:

$$
\begin{aligned}
R[k] & =\sum_{n=(k-1) N}^{k N}\left\{r[n] \bar{c}[n+\bar{\theta}] e^{j\left(2 \pi\left(f_{I F}+\bar{f}_{d_{k}}\right)\right) n T_{S}}\right\}(3) \\
& \triangleq s[k]+w[k]
\end{aligned}
$$

where $\left\lfloor N=T_{c o h} F_{s}\right\rfloor$ denotes the coherent integration time $T_{c o h}$ (s) in samples; $s_{n}[k], w_{n}[k]$ are the signal and the noise components respectively, and [7]

$$
\left\{\begin{aligned}
s[k] & =\sqrt{2 C} N \mathcal{R}[\tau] \operatorname{sinc}\left[\triangle \bar{f}_{d_{k}} T_{c o h}\right] e^{j\left(\pi \triangle \bar{f}_{d_{k}} T_{c o h}+\phi_{k}\right)} \\
& \triangleq G_{n_{k}} e^{j \Phi_{k}} \\
w[k] & =\sum_{n=(k-1) N}^{k N} n_{W}[k+n] \bar{c}[n+\bar{\theta}] e^{j\left[2 \pi\left(f_{I F}+\bar{f}_{d_{k}}\right) n T_{S}\right]}
\end{aligned}\right.
$$


where $(\tau=\theta-\bar{\theta})$ and $\left(\triangle \bar{f}_{d_{k}}=f_{d}-\bar{f}_{d_{k}}\right)$ are the difference between actual and estimated code delays and Doppler shifts at time instance $k$ respectively; $\left(\phi_{k}=2 \pi \triangle \bar{f}_{d_{k-1}} T_{c o h}+\phi_{k-1}\right) ; \Phi_{k}=\pi \triangle \bar{f}_{d_{k}} T_{\text {coh }}+\phi_{k} ;$ $\operatorname{sinc}(x)=\sin (\pi x) / \pi x$; and $\mathcal{R}[\tau]$ is the autocorrelation function.

Then the magnitude $S_{m}=|R[k]|^{2}$ of each complex correlator output is compared with a predetermined threshold $(V)$ to decide which hypothesis between $H_{0}$ $\left(S_{m}<V\right)$ and $H_{1}\left(S_{m}>V\right)$ is true, where $H_{0}$ means absence of the desired signal; and $H_{1}$ means presence of the desired signal. $S_{m}$ is also referred to as the Cross Ambiguity Function (CAF).

If $H_{1}$ happens then the receiver switches from the acquisition to the tracking process to dynamically synchronize with the received signal as well as to demodulate the navigation data. But if $H_{0}$ happens, the acquisition process moves on the next cell of the search-space. After scanning the whole search-space but $H_{1}$ still does not happen then the acquisition process decides that the considered satellite is not in view; and moves on to the next satellite.

\section{FFT Parallel Acquisition Approach}

Let us define $x[n]=r[n] \exp \left(j 2 \pi\left(f_{I F}+\bar{f}_{d_{k}}\right) n T s\right)$, then (3) becomes

$$
R_{n}=\sum_{n=0}^{N-1} x[n] \bar{c}[n+\bar{\theta}]
$$

Equation (5) is the correlation between $x$ and $\bar{c}$, which in fact is very similar to the convolution between them. In [2], based on the similarity between correlation and convolution, it is proved that

$$
\mathbf{R}=\operatorname{DFT}\left\{R_{n}\right\}=(\operatorname{DFT}\{x\})^{*} \operatorname{DFT}\{\bar{c}\}
$$

with DFT $\{$.$\} is the Discrete Fourier Transform of (.) [8].$ The inverse of $\mathbf{R}$ is

$$
R_{n}^{\prime}=\operatorname{IDFT}\{\mathbf{R}\}
$$

$R_{n}^{\prime}$, called the circular correlation of $x$ and $\bar{c}$, is not identical with $R$ because of the periodic property in the time domain of the DFT. However, regarding the fact that $x$ and $\bar{c}$ are composed of periodic PRN sequences, therefore, $R_{n}=R_{n}^{\prime}$.

Basically, DFT can be computed very efficiently by using Fast Fourier Transform (FFT) method [8]. This fact gives the computational advantage to correlation process using FFT:

$$
R_{n}=\operatorname{IFFT}\left\{\operatorname{FFT}\{x\}^{*} \operatorname{FFT}\{\bar{c}\}\right\}
$$

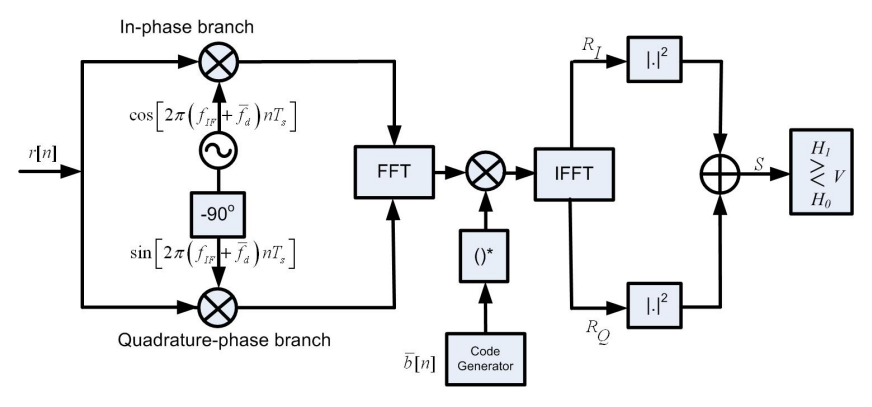

Figugre 3. FFT based acquisition engine: parallels in code delay direction (FFT-time acquisition method)

Figure 3 shows the block diagram of the FFT-time parallel acquisition method $[2,9,10]$. The algorithm can be summarized as follows:

Step 1: Down-convert the $T_{c o h}$-s digital incoming signal by multiplying with a complex signal which already takes into account the tentative value $\bar{f}_{d}$

Step 2: Take FFT of the resulting complex signal.

Step 3: Take the conjugate values of the FFT of the local code $\bar{c}$ with the length equivalent to $T_{c o h}$ s.

Step 4: Take the IFFT of the product of two signals generated at Step 2 and Step 3.

After Step 4, all CAFs values associated with a whole row in Figure 6 are computed.

\section{Impact of code Doppler on signal acquisition}

The Doppler effect observed at the receiver location is caused by the time-variant propagation delay of the transmitted signal along its path toward the receiver [1]. This delay changes over time even in case of a lowdynamics user (e.g. pedestrians, etc.), as at least the SV is moving along its own orbit. This effect changes the real values of the carrier frequency and the code rate of the received signal. The impact on the carrier frequency is taken into account by searching along the Doppler shift uncertainty. The impact on the code rate is also visible, which can be represented as follows:

$$
R_{c}=\tilde{R}_{c}\left(1+\frac{f_{d}}{f_{R F}}\right)
$$

where $\tilde{R}_{c}=1.023$ Mcps is the nominal code rate value.

During the acquisition phase, if the local code is generated at the nominal chip rate $\tilde{R}_{c}$, the correlation between local and received codes suffers a loss due to the difference with the true received chip rate $R_{c}$. The difference between $R_{c}$, and $\tilde{R}_{c}$ leads to a change in the 
spreading code period as it can be seen in Figure 4 where an example of 3 periods of a 4-chips spreading code are represented.

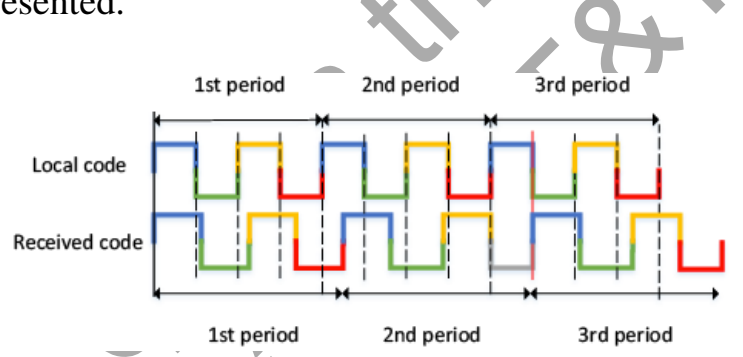

Figugre 4. Code Doppler effect on the spreading code rate. An example of 3 periods, each with 4 chips

That difference also results in the correlation loss as seen in Figure 5. It should be noted that such a loss increases with the integration times. A loss of about 8 $\mathrm{dB}$ in correlation value can be estimated at $C / N_{0}=24$ $\mathrm{dB}-\mathrm{Hz}$ when $T_{i n t}=1 \mathrm{~s}$ as in the weak signal acquisition scenario [11]. Considering the FFT-based signal acquisition method, since the method used a common received signal block of $T_{\text {coh }} \mathrm{s}$ for a whole search space then the code Doppler loss is even worse than in case of the serial search which uses the continuous flow of received signal samples.

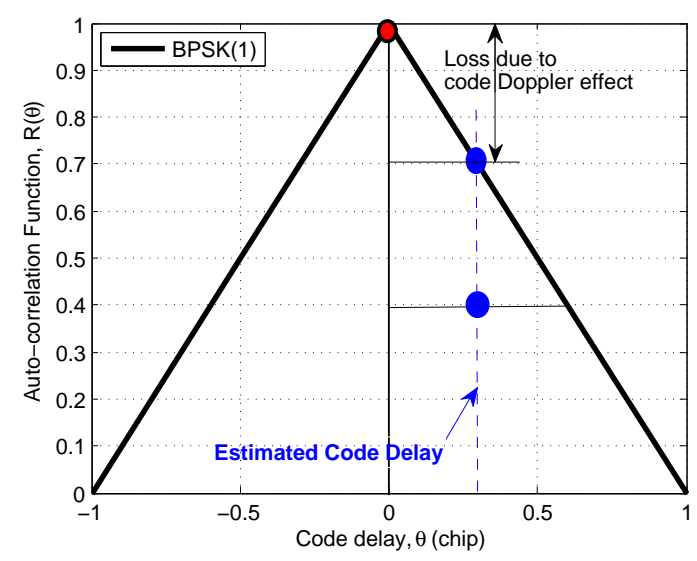

Figugre 5. Correlation loss due to code Doppler effect

\section{Proposed Hybrid Method}

Based on the analysis in Section II, this section introduces a hybrid acquisition method leveraging the advantages of both the conventional serial search and the FFT-time acquisition methods, as well as the compensation mechanism for the code Doppler effect. The proposed method is composed of two steps (see Figure 6):

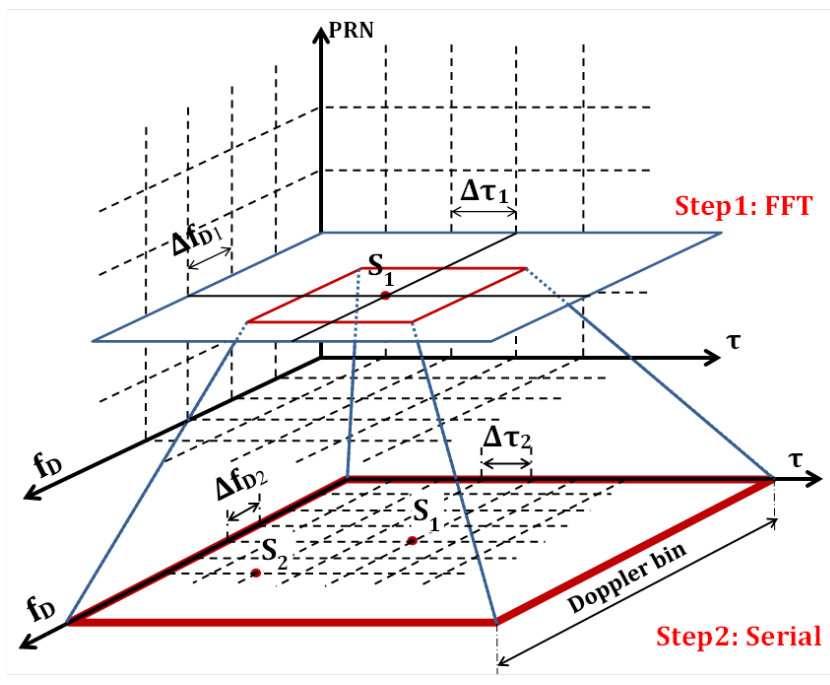

Figugre 6. Search spaces in the proposed method

Step 1 - Rough estimation by using FFT-time: This step leverages the fast nature of the FFT algorithm in order to quickly determine the rough estimations of $\left(\bar{\theta}, \bar{f}_{d}\right)$. The step sizes of the search space are set by the conventional values as in Figure 1. The downsampling process with a factor $K$ might be performed to ease the computational complexity in this step. The estimated cell of this step is denoted as $S_{1}\left(\bar{\theta}_{1}, \bar{f}_{d, 1}\right)$.

Step 2 - Fine estimation by using serial search with code Doppler compensation:

As pointed out in Figure 6, the serial search method tests cells around the rough estimated cell $S_{1}$. This fact results in the new and narrower uncertainties of code delay and Doppler shift: $\left[\bar{\theta}_{1}-m, \bar{\theta}_{1}+m\right]$ chips; $\left[\bar{f}_{d, 1}-\right.$ $\left.h, \bar{f}_{d, 1}+h\right] \mathrm{Hz}$. With the step sizes of Step-1, the rough estimations of Step-1 cannot be more than one cell off the correct one (see Figure 1), therefore, $m=2$ chips and $h=500 \mathrm{~Hz}$.

In addition, to take into account the code Doppler effect, the local code is generated with the code rate $R_{c}$ being:

$$
R_{c}=1.023\left(1+\frac{\bar{f}_{d, 1}}{f_{R F}}\right) \text { Mcps }
$$

The search-space step sizes used in this step are reduced to refine the estimation as follows:

- the code delay step size equals to the sampling period $T_{S}$, i.e. $\triangle \theta_{2}=T_{S} \times R_{c}$ chip;

- the Doppler step size equals to half of that at Step-1, i.e. $\triangle f_{D, 2}=0.5 \triangle f_{D, 1}=250 \mathrm{~Hz}$.

This fact of Step-2 also results in reducing the needed 
time for the signal tracking, the process after the acquisition, to be locked.

With this configuration of Step-2, if $F_{S}=16.368$ $\mathrm{MHz}$, the number of rows, and the number of columns of the refined search space are $N_{f, 2}=3$, and $N_{c, 2}=35$, respectively.

\section{Performance analysis}

In this section, the proposed hybrid acquisition method is analysed with the real signal collected from the GPS constellations via an antenna located at the roof of the Ta Quang Buu library building at Hanoi University of Science and Technology (see Figure 7). The radio frequency signal from the antenna goes through the frontend module, which is responsible for conditioning and converting the analog radio frequency signals to the digital intermediate frequency (IF) samples. In this campaign, the configuration of the frontend module is $F_{S}=16.368 \mathrm{MHz}, f_{I F}=4.092 \mathrm{MHz}$, and $B_{w}=4.2$ $\mathrm{MHz}$.

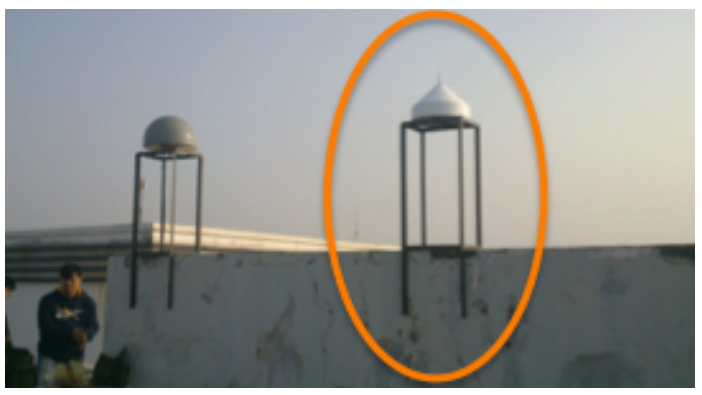

Figugre 7. The location of the antenna in the campaign

\section{A. Estimation quality of the proposed hybrid method}

Figure 8(a) shows the search space after Step-1. As mentioned in Section III, the search space sizes are $\triangle \theta_{1}=0.5$ chip, and $\triangle f_{D, 1}=500 \mathrm{~Hz}$. The estimated cell $S_{1}$, which is the highest peak of the search space, is

$$
\left\{\begin{array}{l}
\bar{\theta}_{1}=758.5 \text { chips }=12136 \text { samples } \\
\bar{f}_{d, 1}=0 \mathrm{~Hz}
\end{array}\right.
$$

$S_{1}$ is then fed to Step-2 for finer estimations.

In Step-2, $\triangle \theta_{2}=0.0625$ chip $=1$ sample, and $\triangle f_{D, 2}=250 \mathrm{~Hz}$. As seen in Figure 8(b), the estimated cell $S_{2}$ is

$$
\left\{\begin{array}{l}
\bar{\theta}_{2}=12141 \text { samples } \\
\bar{f}_{d, 2}=0 \mathrm{~Hz}
\end{array}\right.
$$

The estimation differences between the two steps are

$$
\left\{\begin{array}{l}
\delta \theta=\bar{\theta}_{1}-\bar{\theta}_{2}=5 \text { samples } \\
\delta f_{D}=\bar{f}_{d, 2}-\bar{f}_{d, 2}=0 \mathrm{~Hz}
\end{array}\right.
$$

As seen in Figure 9, these improvements provided by Step-2 give a processing gain, or also referred to as the acquisition sensitivity improvement, $G$ :

$$
G=10 \lg \left(\frac{164,903,765}{88,312,483}\right) \approx 2.7 \mathrm{~dB}
$$

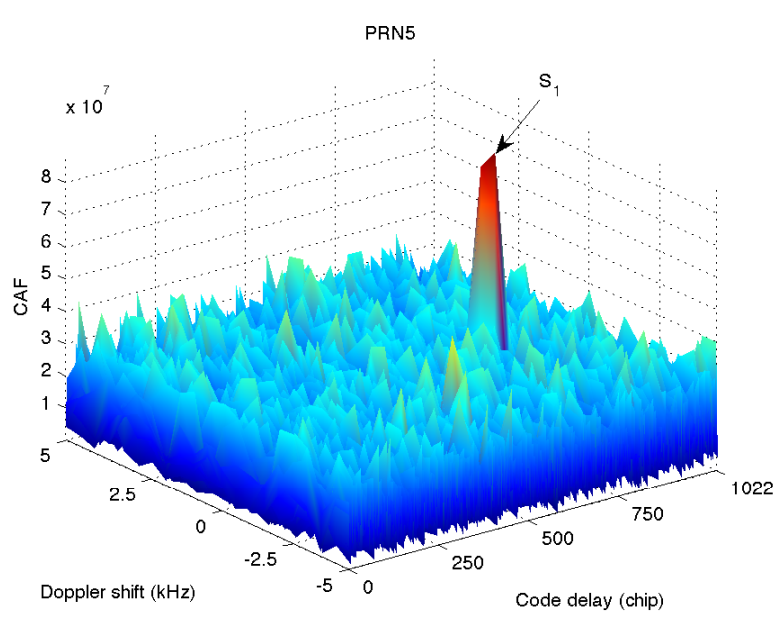

(a)

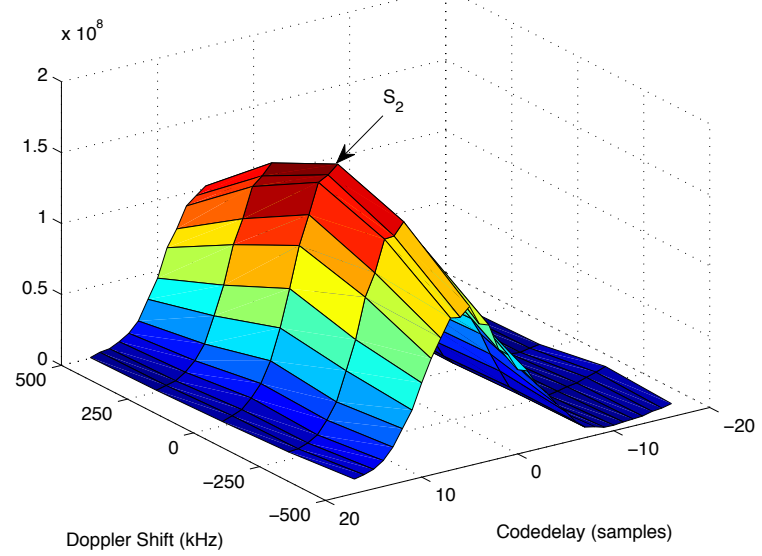

(b)

Figugre 8. Acquisition result of the hybrid method: (a) Step-1: rough estimations; (b) Step-2: fine estimations

It should be noted that Step-1 and the conventional serial search (Section II-B) are similar in term of the estimation quality, since the search space sizes are set similarly. Therefore, the $2.7 \mathrm{~dB}$ gain is also the advantage of the proposed hybrid method over the conventional serial search.

\section{B. Complexity concerns}

In this section, the computational complexity is analysed among the three methods, namely: (i) Conventional 
Table I. Complexity comparison among different methods

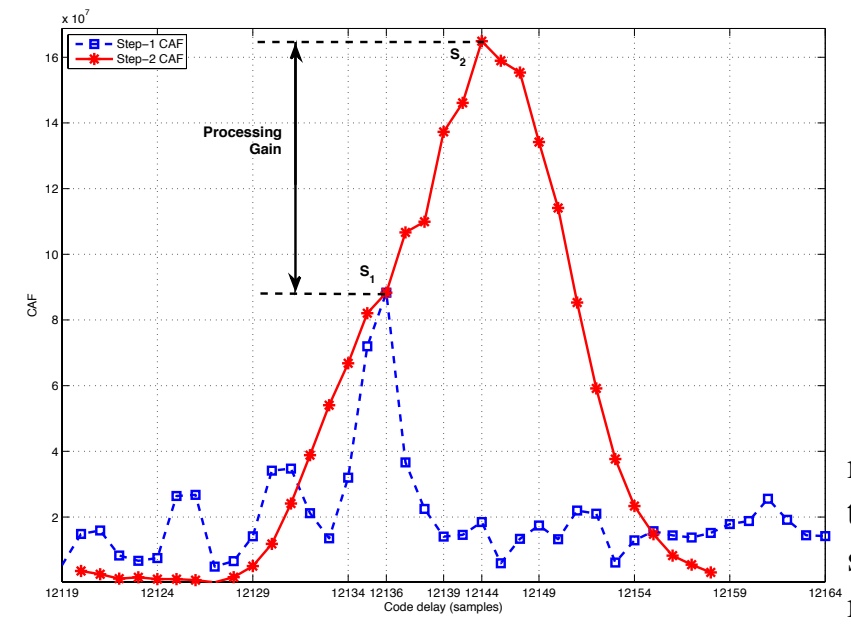

Figugre 9. Processing gain given by the finer estimations of Step-2

serial search; (ii) FFT-time search; (iii) Proposed hybrid method. Table I reports the comparison of the execution time of the three methods. As pointed out in Section II-A, the search space has $N_{f}=21$ rows, $N_{c}=2046$ columns, and $L=42,966$ cells. The execution time here is defined as a needed duration for a cell to be tested, therefore:

- For serial search: the execution time is denoted as $t_{S}$, and after each $t_{S}$ seconds a cell of the search space is tested. With the number of cells in the search space is $L$, the number of tests (or number of repetitions) for the serial search is also $L$. Then the required time for a whole search space to be tested is: $\mathcal{T}_{\mathcal{S}}=t_{S} \times L$.

- For FFT-time parallel search: the execution time is denoted as $t_{F}$, and after each $t_{F}$ seconds, a whole row of the search space (i.e. all possible code delays, see Figure 1) is tested. Therefore, for a whole search space to be tested, the needed time is $\mathcal{T}_{\mathcal{F}}=t_{F} \times N_{f}$;

- For proposed hybrid search: with this method, two steps are performed, therefore the needed time for the acquisition process is $\mathcal{T}_{\mathcal{H}}=\mathcal{T}_{1}+\mathcal{T}_{2}$; with $\mathcal{T}_{1}=$ $\mathcal{T}_{\mathcal{F}}$ and $\mathcal{T}_{2}=t_{S} \times\left(N_{f, 2} \times N_{c, 2}\right)$, where $N_{f, 2}=3$, and $N_{c, 2}=35$ (see Section III).

Table I reports the execution times, which are measured using the tic and toc functions of Matlab, run on a normal PC (2.53 GHz Intel Core 2 Duo). The results show that the proposed hybrid method has a comparable computational speed with the FFT-time acquisition but much faster than the conventional serial one.

\begin{tabular}{|l|c|c|r|}
\hline Method & $\begin{array}{c}\text { Execution } \\
\text { Time (s) }\end{array}$ & $\begin{array}{c}\text { Repetition } \\
\text { (times) }\end{array}$ & Total (s) \\
\hline Serial (i) & 0.0042 & 42966 & 180.45 \\
FFT-time (ii) & 0.0056 & 21 & 0.11 \\
Hybrid (iii) & & $21(\mathrm{ii})+105(\mathrm{i})$ & 0.59 \\
\hline
\end{tabular}

\section{Conclusion}

This paper introduced a hybrid signal acquisition method which leverages the advantages of the conventional serial search acquisition and the FFT-based parallel search one. Two steps are performed in the proposed method before the parameters of the received signal are derived. The performance analyses proves a significant improvement in the processing gain, or the acquisition sensitivity, of the proposed method over the conventional one, meanwhile the computational complexity is kept comparable to that of the FFT-based parallel one.

\section{REFERENCES}

[1] E. D. Kaplan, Ed., Understanding GPS: Principles and Applications, 2nd ed. Artech House, November 2005.

[2] J. B.-Y. Tsui, Fundamentals of Global Positioning System Receivers: a Software Approach, 2nd ed. Wiley-Interscience, 2005.

[3] T. H. Ta, "Acquisition Architecture for Modern GNSS Signals," Ph.D. dissertation, Polytechnique University of Turin, Italy, 2010.

[4] "Navstar GPS Interface Specification ISGPS-200 revision D," Navstar GPS Joint Program Office, Tech. Rep., March 2006. [Online]. Available: http://www.navcen.uscg.gov/ gps/modernization/IRN-200D-001\%207Mar06.pdf

[5] T. H. Ta, N. C. Shivaramaiah, A. Dempster, and Let, "Significance of Cell-correlation Phenomenon in GNSS Matched Filter Acquisition Engines," IEEE Transactions on Aerospace and Electronic Systems, to be published.

[6] T. H. Ta, S. U. Qaisar, A. Dempster, and F. Dovis, "Partial Differential Post Correlation Processing for GPS L2C Signal Acquisition," IEEE Transactions on Aerospace and Electronic Systems, vol. 8, no. 2, April 2012.

[7] J. K. Holmes, Ed., Spread Spectrum Systems for GNSS and Wireless Communications. Artech House, 2007.

[8] A. V. Oppenheim, Ed., Signals and Systems, 2nd ed. Prentice-Hall, 1996. 
[9] D. J. R. V. Nee and A. J. R. M. Coenen, "New fast gps code-acquisition technique using FFT," Electronics Letters, vol. 27, no. 2, pp. 158-160, 1991.

[10] D. J. R. Van Nee and A. J. R. M. Coenen, "Novel fast GPS / GLONASS code acquisition technique using low update rate Fast Fourier Transform," Electronics Letters, vol. 28, no. 9, pp. 863-865, 1992

[11] F. Dovis and T. H. Ta, "Chapter 1: High Sensitivity Techniques for GNSS Signal Acquisition, , InTech Publisher, February 2012, Global Navigation Satellite Systems: Signal, Theory and Applications, Prof. Shuanggen Jin (Ed.)," InTech Publisher, 2012.

\section{AUTHOR'S BIOGRAPHY}

Tung Hai Ta received his $\mathrm{PhD}$ degree in Information and Communication Technologies (2/2010) from Politecnico di Torino (PoliTo), Italy. In 2009, he was with Satellite Navigation and Positioning Laboratory (SNAP Lab), University of New South Wales. From 2/2010 to 2/2011, he worked as a PostDoc fellow at Electronics Department, PoliTo. Since 3/2011, he has worked in School of Information and Communication Technology, HUST. Since 12/2011, he has been the Director of the International Centre for Research and Development of Satellite Navigation Technology in South-East Asia (NAVIS Centre), Hanoi, Vietnam. His research activities are focused on GNSS signal processing and Nav/Com integration technologies. 